\title{
The Lactulose Breath Test in Irritable Bowel Syndrome: Is It All Hot Air?
}

\author{
Rebecca E. Burgell ${ }^{1}$ Peter R. Gibson ${ }^{1}$
}

Published online: 7 November 2015

(c) Springer Science+Business Media New York 2015

The microbial ecosystem that occupies the human intestine is essential for the maintenance of health. An intricate relationship exists between the gut microbiome, the central nervous system, the neuroimmune and neuroendocrine systems, the autonomic nervous system, and the enteric nervous system, the interplay of which is responsible for the effective control of the gut by the higher centers.

The development of new techniques that enable better characterization of gut bacteria has noticeably increased interest how the human microbiome affects the development of disease, particularly so in the pathophysiology of functional gastrointestinal disorders, where epidemiological studies have long-linked acute gut infection with the development of chronic functional symptoms. Up to $30 \%$ of patients develop irritable bowel syndrome (IBS) after suffering from a gastrointestinal infection, and $40 \%$ describe symptomatic benefit from antibiotic therapy [1]. IBS has also been associated with altered diversity and composition of gut bacteria [2]. Although the exact mechanisms remain poorly defined, the gut microbiome is firmly associated with IBS pathogenesis.

Given the presumed pathogenic role of the gut microbiota in IBS, its manipulation is now a "hot" topic in management, from the addition of "good bacteria" via probiotics, the promotion of "good bacteria" via prebiotics, the change of bacterial populations by antibiotics, and the replacement of bacteria via fecal microbiota transfer. Antibiotics have received the most attention since

Peter R. Gibson

peter.gibson@monash.edu

1 Department of Gastroenterology, Alfred Hospital, Monash University, 99 Commercial Road, Melbourne, VIC 3004, Australia they are a major therapeutic tool in treating small intestinal bacterial overgrowth (SIBO), postulated to be an important link between gut bacteria and IBS. The small bowel normally contains only a fraction of the bacteria present in the large bowel $\left(10^{4}-10^{8}\right.$ vs. $\left.10^{9}-10^{12}\right)$ [3]. SIBO, defined as an excess of colonic-type bacteria in the small bowel, is often diagnosed in patients with small bowel abnormalities such as altered anatomy, motility, or flow resulting in stasis, enhancing bacterial proliferation. While SIBO provides an attractive (and potentially treatable) cause for the symptoms of IBS, in reality diagnosing SIBO in IBS patients who have normal anatomy is fraught with controversy.

The major issue associated with this concept is the lack of a "gold standard" to achieve diagnostic accuracy. Since 4-78\% of tested patients with IBS have SIBO [4], this large disparity exists as a result of the variety of diagnostic techniques available for its diagnosis. Culture and genomic techniques are limited by the sampling location (proximal vs. distal small bowel), by potential contamination, and by the site of bacterial overgrowth (luminal liquid vs. adherent biofilm). Given these concerns, indirect measures of bacterial function and location are often utilized clinically. Again, these are not without limitations: An increase in hydrogen concentration in the exhaled breath following glucose administration has high specificity for diagnosing SIBO, but poor sensitivity, as glucose is rapidly absorbed in the small bowel and hence is useful to diagnose proximal gut overgrowth only unless transit is very rapid. Breath hydrogen responses following ingestion of the disaccharide lactulose, which is resistant to hydrolysis by mammalian saccharidases, are thus more commonly used to diagnose SIBO in patients with functional gastrointestinal disorders.

Support for its clinical use comes from encouraging reports that indicate that the presence of SIBO as diagnosed 
by lactulose breath testing (LBT) is associated with an improvement in symptoms following antibiotics in $48 \%$ of patients with IBS [5], which corresponds with normalization of LBT, and that the use of use of serial LBT (until normalization) is useful to determine the duration of antibiotic therapy [6]. Such positive data and opinion must be weighed against the poor correlation of LBT-diagnosed SIBO with that detected with culture techniques [4]; the reported overall sensitivity of LBT in the detection of SIBO is $17-68 \%$, with a specificity of $44-86 \%$ [7]. Such figures are of course flawed in the absence of a widely accepted "gold standard."

Given the controversy as to whether LBT can effectively diagnose SIBO in IBS in the first place, perhaps a more useful outcome from LBT would be to predict therapeutic response to treatments such as antibiotics regardless of the presence of SIBO. Such an approach was taken by Kisir et al. [8] who in this issue of Digestive Diseases and Sciences retrospectively examined clinical responses to a diverse range of antibiotics in a large population of 561 patients with IBS who had a LBT. While the definition of response was a somewhat vague "global physician assessment" and the duration of follow-up was not stated, a response of $80 \%$ overall is impressive, even in this high placebo-responding population. It seemed that patients were treated with antibiotics if they had features previously attributed to SIBO via a LBT or in those whose LBT was non-informative (i.e., an inadequate rise of hydrogen and/ or methane). The 152 patients not treated presumably had what might be loosely regarded as a "normal" test result. This was not clear in the report. The authors classified the LBT findings into four groups-(1) the non-informative, (2) those with an early rise that started to fall before $90 \mathrm{~min}$, (3) those with a very high peak ( $\geq 50 \mathrm{ppm}$ ), or (4) those with miscellaneous patterns that were not described. This then enabled a retrospective examination of the response to antibiotics. Interestingly, those with non-informative tests were the most predictive (or informative!). In other words, a "poor" response in breath hydrogen and/ or methane to lactulose was associated with a $95 \%$ symptomatic response to antibiotics in contrast to, for example, a high peak level (a "type 3" response?) where a significantly lower proportion (47\%) responded.

What do such findings tell us with regard to the LBT? The authors state that this "contradicts the classic definition of a positive LBT." Unfortunately, the so-called classical features themselves are poorly supported by evidence. The early rise in breath hydrogen/methane is frequently secondary to rapid gut transit rather than SIBO as demonstrated by concurrent LBT and oro-cecal scintigraphy [9]. The high peak breath hydrogen/methane has little supporting diagnostic evidence. It is unfortunate that a highly predictive pattern such as a high basal breath hydrogen was not applied in the current study, since it may be a good indicator of at least proximal SIBO, as confirmed by jejunal fluid culture studies. Other patterns like a double peak have been debunked as they also are observed in some healthy subjects [3].

What do the findings tell us about clinical decisions to use antibiotics? As pointed out by the authors, widespread use of antibiotics in a common condition like IBS is environmentally irresponsible. Nevertheless, targeting antibiotic therapy to a highly responsive group is needed. Perhaps the low hydrogen/methane producers provide such a clue. Yet, the clinical data provided in this retrospective study cannot inform such a decision since IBS is a chronic condition in which acute benefits may not influence longerterm outcomes.

What the study does tell us is that criteria being applied to indicate a high chance of the presence of SIBO in IBS subjects are questionable, supporting other evidence of poor performance characteristics of the LBT. The data do not tell us that the LBT should be used to identify patients for antibiotic therapy as even the worst performing groups had a near $50 \%$ response rate and those with "normal" LBT findings were not treated. Perhaps the recommendation from a recent systematic review that a "test, treat, and outcome assessment" strategy is most appropriate when managing potential SIBO in patients with IBS [10] remains the best clinical strategy if antibiotics are going to be used. It is important that the many limitations of hydrogen breath tests and its interpretation are understood. Using a test where the non-informative is most informative is an unattractive option.

\section{Compliance with ethical standards}

Conflict of interest None.

\section{References}

1. Pimentel M, Lembo A, Chey WD, et al. Rifaximin therapy for patients with irritable bowel syndrome without constipation. $N$ Engl J Med. 2011;364:22-32.

2. Jeffery IB, O'Toole PW, Ohman L, et al. An irritable bowel syndrome subtype defined by species-specific alterations in faecal microbiota. Gut. 2012;61:997-1006.

3. Gibson PR, Barrett JS. The concept of small intestinal bacterial overgrowth in relation to functional gastrointestinal disorders. Nutrition. 2010;26:1038-1043.

4. Ghoshal UC, Srivastava D, Ghoshal U, et al. Breath tests in the diagnosis of small intestinal bacterial overgrowth in patients with irritable bowel syndrome in comparison with quantitative upper gut aspirate culture. Eur J Gastroenterol Hepatol. 2014;26:753-760.

5. Pimentel M, Chow EJ, Lin HC. Eradication of small intestinal bacterial overgrowth reduces symptoms of irritable bowel syndrome. Am J Gastroenterol. 2000;95:3503-3506.

6. Bae S, Lee KJ, Kim YS, et al. Determination of rifaximin treatment period according to lactulose breath test values in 
nonconstipated irritable bowel syndrome subjects. J Korean Med Sci. 2015;30:757-762.

7. Saad RJ, Chey WD. Breath testing for small intestinal bacterial overgrowth: maximizing test accuracy. Clin Gastroenterol Hepatol. 2014;12:1964-1972.

8. Kasir R, Zakko S, Zakko P, et al. Predicting a response to antibiotics in patients with the irritable bowel syndrome. Dig Dis Sci. (Epub ahead of print). doi:10.1007/s10620-015-3872-9.
9. Yu D, Cheeseman F, Vanner S. Combined oro-caecal scintigraphy and lactulose hydrogen breath testing demonstrate that breath testing detects oro-caecal transit, not small intestinal bacterial overgrowth in patients with IBS. Gut. 2011;60:334-340.

10. Khoshini R, Dai SC, Lezcano S, et al. A systematic review of diagnostic tests for small intestinal bacterial overgrowth. Dig Dis Sci. 2008;53:1443-1454. 